University of Wollongong

Research Online

Australian Institute for Innovative Materials -

Papers

Australian Institute for Innovative Materials

2009

The role of optical rectification in the generation of terahertz radiation from

GaBiAs

Krunal Radhanpura

University of Wollongong, krunal@uow.edu.au

Stuart Hargreaves

Roger A. Lewis

University of Wollongong, roger@uow.edu.au

M Henini

Follow this and additional works at: https://ro.uow.edu.au/aiimpapers

Part of the Engineering Commons, and the Physical Sciences and Mathematics Commons

Research Online is the open access institutional repository for the University of Wollongong. For further information contact the UOW Library: research-pubs@uow.edu.au 


\title{
The role of optical rectification in the generation of terahertz radiation from GaBiAs
}

\author{
Abstract \\ We report on a detailed study of the emission of terahertz-frequency electromagnetic radiation from

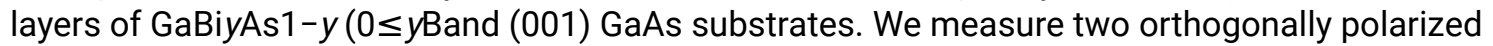 \\ components of the terahertz radiation emitted under excitation by ultrashort near-infrared laser pulses in \\ both transmission and reflection geometries as a function of the crystal rotation about its surface normal \\ as well as the effect of in-plane magnetic field and pump fluence on the terahertz emission. We conclude \\ that the principal mechanism for terahertz generation is via optical rectification rather than transient \\ currents.

\section{Disciplines} \\ Engineering | Physical Sciences and Mathematics

\section{Publication Details} \\ Radhanpura, K., Hargreaves, S., Lewis, R. A. \& Henini, M. (2009). The role of optical rectification in the \\ generation of terahertz radiation from GaBiAs. Applied Physics Letters, 94 (25), 251115-1-251115-3.
}




\title{
The role of optical rectification in the generation of terahertz radiation from GaBiAs
}

\author{
K. Radhanpura, ${ }^{1}$ S. Hargreaves, ${ }^{1}$ R. A. Lewis, ${ }^{1, a)}$ and M. Henini ${ }^{2}$ \\ ${ }^{1}$ Institute for Superconducting and Electronic Materials, University of Wollongong, Wollongong, \\ New South Wales 2522, Australia \\ ${ }^{2}$ School of Physics and Astronomy, and Nottingham Nanotechnology and Nanoscience Centre, \\ University of Nottingham, NG7 2RD Nottingham, United Kingdom
}

(Received 27 April 2009; accepted 2 June 2009; published online 26 June 2009)

\begin{abstract}
We report on a detailed study of the emission of terahertz-frequency electromagnetic radiation from layers of $\mathrm{GaBi}_{y} \mathrm{As}_{1-y}(0 \leq y<0.04)$ grown by molecular beam epitaxy on $(311) B$ and $(001) \mathrm{GaAs}$ substrates. We measure two orthogonally polarized components of the terahertz radiation emitted under excitation by ultrashort near-infrared laser pulses in both transmission and reflection geometries as a function of the crystal rotation about its surface normal as well as the effect of in-plane magnetic field and pump fluence on the terahertz emission. We conclude that the principal mechanism for terahertz generation is via optical rectification rather than transient currents. (C) 2009 American Institute of Physics. [DOI: 10.1063/1.3157272]
\end{abstract}

The substitution of Bi for As in GaAs (Refs. 1-5) results in a dramatic decrease in the band gap, $\sim 90 \mathrm{meV}$ per percent $\mathrm{Bi}$. For this reason $\mathrm{GaBiAs}$ is attracting attention for long wavelength $(>1 \mu \mathrm{m})$ photonics applications including terahertz optoelectronics.

Time-resolved terahertz spectroscopy has been used to study charge carrier dynamics in GaBiAs. The electron mobility $\mu_{e}$ was found to be not significantly reduced with incorporation of $\mathrm{Bi}$ up to the level of $1.4 \% .^{6}$ Other studies ${ }^{7,8}$ have identified a double exponential decay, both decays on picosecond timescales, corresponding respectively to electron trapping and trap emptying. Dipole antennas fabricated on $\mathrm{GaBiAs}$ have been demonstrated to be effective detectors of terahertz radiation ${ }^{9,10}$ with a spectral width $>4 \mathrm{THz}$. GaBiAs photoconductive terahertz emitters have also been fabricated, permitting the realization of an all-GaBiAs terahertz time-domain spectroscopy (TDS) system. ${ }^{11}$

The earlier work on GaBiAs as a terahertz emitter has been restricted to the photoconductivity mechanism. However, terahertz radiation is known to be emitted from semiconductors by other mechanisms such as by transient currents (TC) — which arise from surface-field effects and the photo-Dember effect - and by optical rectification (OR) which includes bulk and surface (electric-field-induced) OR. In this letter we explore the importance of these mechanisms other than photoconductivity in the emission of terahertz radiation from GaBiAs.

The growth by molecular beam epitaxy and the characterization by high-resolution $\mathrm{x}$-ray diffraction, transmission electron microscopy, Z-contrast imaging, and optical transmission spectroscopy of the samples used in this study have been detailed previously. ${ }^{12}$ For each of the orientations (001) and $(311) B$, four $\mathrm{GaBi}_{y} \mathrm{As}_{1-y}$ layers corresponding to four different As fluxes during growth (see Fig. 2 of Ref. 12) as well as a control layer of $\mathrm{GaAs}(y=0)$ and the virgin substrate were examined, a total of 12 samples in all. The GaBiAs epilayer thickness in all cases was nominally $1 \mu \mathrm{m}$.

a) Electronic mail: roger@uow.edu.au.
Optical excitation was by sub-12-fs pulses of horizontally (H-)polarized radiation. Since the absorption coefficient of GaAs at the near-infrared (NIR) excitation laser center wavelength of $790 \mathrm{~nm}$ (frequency $379 \mathrm{THz}$, photon energy $1.57 \mathrm{eV})$ is $\alpha_{\mathrm{NIR}}=1.2 \times 10^{4} \mathrm{~cm}^{-1}$, most of the interaction is with the epilayer, not the substrate. It is possible that there is a terahertz generation mechanism at the interface between the epilayer and the substrate. Before being examined as terahertz emitters, all the samples were tested for terahertz transmission in a conventional terahertz TDS system. As expected, all were found to transmit terahertz radiation with little attenuation. These measurements indicate that the charge-carrier concentration, both in the layers and the substrate, is not large and confirm that terahertz radiation generated in the layers on the front of the sample may travel with little loss through to the back of the sample.

Two experimental geometries were employed to investigate the samples as emitters. In transmission, the excitation beam was perpendicular to the sample and the radiated terahertz field was detected in the straight-through direction. In reflection, the excitation beam was at an angle of incidence of $45^{\circ}$ and the radiated terahertz field was detected in the specular reflection direction. A wire-grid polarizer was used to distinguish $\mathrm{H}$ - and vertically $(\mathrm{V}-)$ polarized components of the terahertz radiation. (In the reflection geometry, $\mathrm{H}$-polarized radiation corresponds to $p$-polarized radiation and V-polarized radiation corresponds to $s$-polarized radiation.) The terahertz radiation was detected electro-optically in free space using a 1-mm-thick (110) ZnTe wafer.

Figure 1 shows, in the time domain, the terahertz field emitted by a (311) $B \mathrm{GaBi}_{0.035} \mathrm{As}_{0.965}$ sample in transmission geometry. The variation in signal before the main peak indicates the level of noise in the system. The spectrum was collected under ambient conditions and the modulation after the main peak is largely due to atmospheric water vapor absorption. In the transmission geometry, all the (311)B samples including the substrate and the control $(y=0)$ sample gave similar time-domain spectra; however, except for one sample $\left(\mathrm{GaBi}_{0.026} \mathrm{As}_{0.974}\right)$, none of the (100) samples gave a discernible signal. This striking contrast immediately sug- 


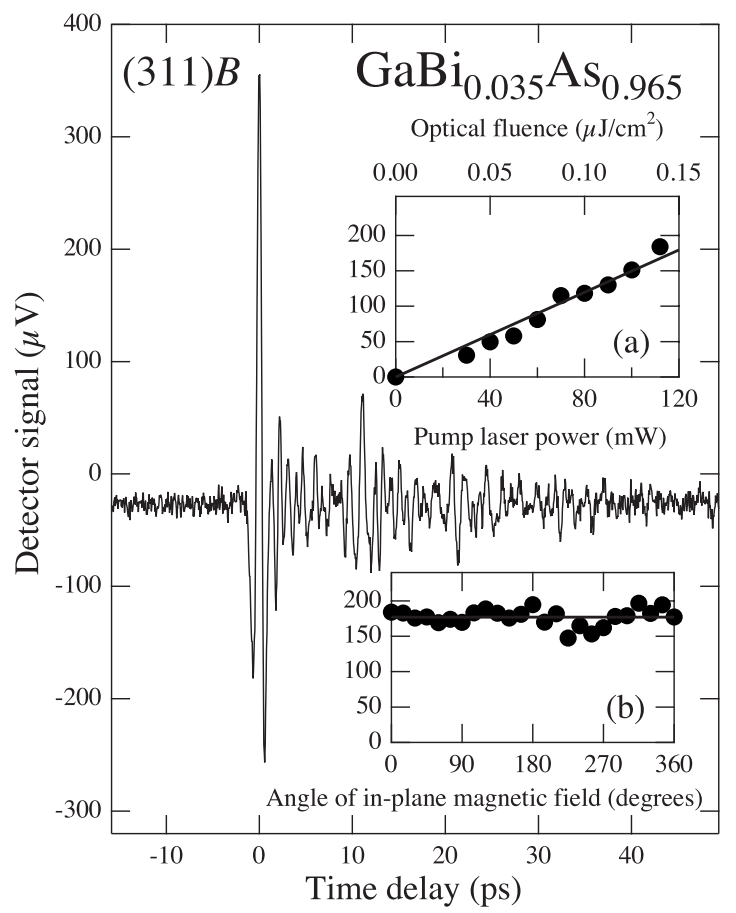

FIG. 1. Time-domain spectrum of terahertz field radiated from (311) $B$ $\mathrm{GaBi}_{0.035} \mathrm{As}_{0.965}$ sample (transmission geometry). (a) Dependence of peak terahertz field on optical fluence of pump beam (reflection geometry). (b) Dependence of peak terahertz field on angle of in-plane magnetic field (reflection geometry).

gests that the terahertz emission such as shown in Fig. 1 is related to the orientation of the pump-beam polarization relative to the principal crystal axes, which is characteristic of $\mathrm{OR}$ and that the quality of the samples is so high with respect to recombination times that they yield little terahertz radiation from TC.

To further explicate the mechanism responsible for the terahertz emission, the dependence of the terahertz field on optical pump fluence was investigated, in reflection geometry, Fig. 1(a). The terahertz field is seen to increase steadily with optical fluence. At low fluences, this is the expected behavior for both TC and OR mechanisms. At high fluences, the TC effect is expected to saturate due to surface-field screening. We see no evidence of saturation and so no evidence of TC mechanisms (although the fluences employed here may be too small for surface-field screening to be significant).

TC effects are influenced by an in-plane magnetic field, which serves to rotate the radiating dipole and so increase or decrease the radiated terahertz field. In rotating the magnetic field through $360^{\circ}$, one maxima and one minimum in the terahertz signal will be observed. ${ }^{13}$ In Fig. 1(b) we show our results when a permanent magnet was rotated behind the sample in reflection geometry. The magnitude of the in-plane magnetic field was $\sim 0.15 \mathrm{~T}$. Within experimental error there is no variation in the radiated terahertz field with respect to in-plane magnetic field angle. This adds further weight to the hypothesis that TC effects play a negligible role in these samples.

The variation of terahertz field with azimuthal angle as the sample is rotated about its normal is the litmus test for OR. In Fig. 2 we show the azimuthal angle dependence of terahertz radiation emitted in the transmission geometry by (a) the (311) $B_{\text {substrate and (b) the (311)B GaBi }}{ }_{0.035} \mathrm{As}_{0.955}$

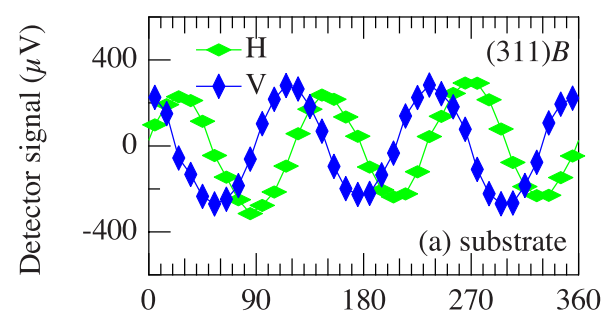

Azimuthal angle (degrees)

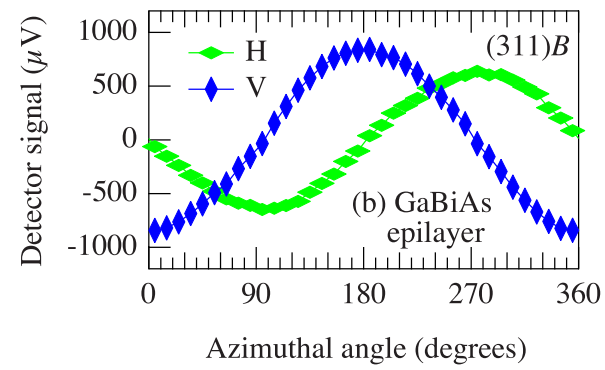

FIG. 2. (Color online) Variation of the peak terahertz field, for both $\mathrm{H}$ - and $\mathrm{V}$-polarizations, as a function of angle of rotation around the sample normal in transmission geometry for $(311) B$ (a) substrate and (b) $\mathrm{GaBi}_{0.035} \mathrm{As}_{0.965}$ epilayer.

sample. All the other layers grown on (311) $B$ substrates including the GaAs control sample showed a similar azimuthal angle dependence to that depicted in Fig. 2(b). We note that both panels of Fig. 2 show a very distinct variation in both $\mathrm{H}$ and $\mathrm{V}$ components of the terahertz radiation, and further that the dependences in the two panels are quite different, involving three maxima per rotation in Fig. 2(a) and one maximum in Fig. 2(b).

We now account for the data of Fig. 2 as arising from OR. The theory of the second-order nonlinear effect of bulk OR has been given in many works ${ }^{14-18}$ for the simple crystallographic faces (100), (110), (111), and more recently ${ }^{19}$ for (112). Expressed in terms of the principal axes of the crystal, the polarization vector $P$ is related to the components of the optical field $E$ through a single component, $d_{14}$, of the susceptibility tensor. The theory of the third-order nonlinear effect of (surface) electric-field-induced OR has also been given $^{20}$ for (100), (110), (111), and ${ }^{19}$ (112) faces. The field $F$ is presumed to be normal to the surface. We introduce $\alpha^{\prime}=3 \chi_{z x x z}\left(E_{x}^{2}+E_{y}^{2}+E_{z}^{2}\right), \quad \beta^{\prime}=6 \chi_{z z x x}\left(E_{x} F_{x}+E_{y} F_{y}+E_{z} F_{z}\right)$, and $\gamma^{\prime}=3\left(\chi_{z z z z}-\chi_{z x x z}-2 \chi_{z z x x}\right)$, where the $\chi^{\prime}$ 's are components of the susceptibility tensor. Then

$$
\begin{aligned}
& P_{x}=2 d_{14} E_{y} E_{z}+\alpha^{\prime} F_{x}+\beta^{\prime} E_{x}+\gamma^{\prime} E_{x}^{2} F_{x}, \\
& P_{y}=2 d_{14} E_{z} E_{x}+\alpha^{\prime} F_{y}+\beta^{\prime} E_{y}+\gamma^{\prime} E_{y}^{2} F_{y}, \\
& P_{z}=2 d_{14} E_{x} E_{y}+\alpha^{\prime} F_{z}+\beta^{\prime} E_{z}+\gamma^{\prime} E_{z}^{2} F_{z} .
\end{aligned}
$$

We now extend the theory to the (311) face studied here. Figure 3(a) is our calculation of the variation in the $\mathrm{H}$ and $\mathrm{V}$ components of the terahertz radiated field as a function of azimuthal angle due to the bulk OR $(F=0)$. Figure $3(\mathrm{~b})$ gives the result for (surface) electric-field-induced OR $\left(d_{14}=0\right.$; the terms involving $\alpha^{\prime}$ and $\beta^{\prime}$ do not vary with azimuthal angle and have also been set to zero). Figure 3(c) gives the result when the bulk and surface effects are combined with the ratio $d_{14}=-4.7 \gamma^{\prime} F$. 

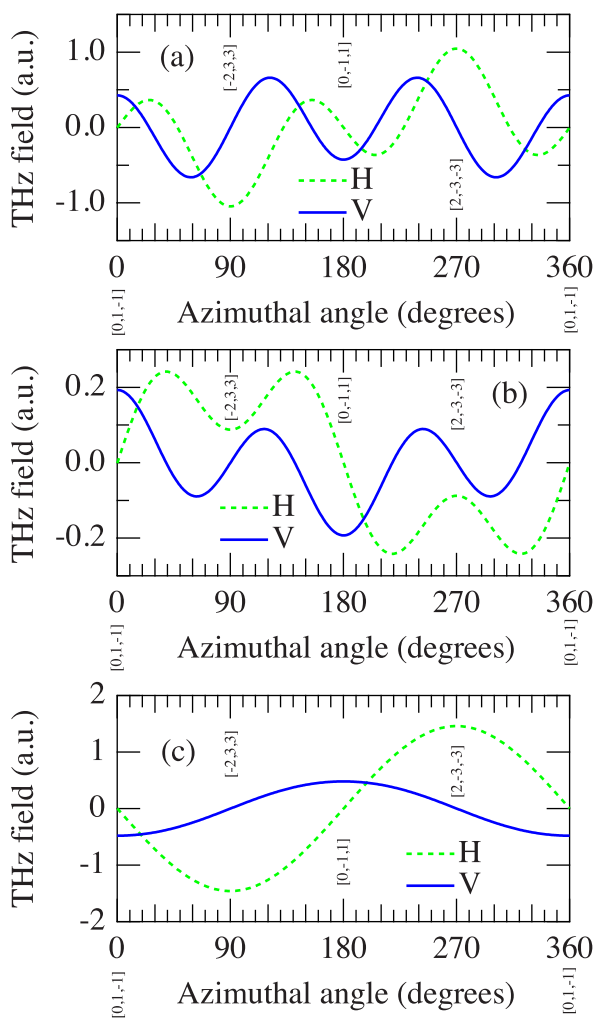

FIG. 3. (Color online) Calculation of H- and V-polarized terahertz fields from a (311) crystal face due to (a) bulk OR, (b) surface electric-fieldinduced OR, and (c) the addition of these using the ratio $d_{14}=-4.7 \gamma^{\prime} F$. The azimuthal angle is measured starting from the optical field being parallel to the $[01 \overline{1}]$ crystallographic direction and increasing in the direction of [2 33$]$.

The data from the substrate, Fig. 2(a), display three maxima in both $\mathrm{H}$ and $\mathrm{V}$ components. On closer examination it is seen that for the $\mathrm{H}$ component, one maximum is slightly higher than the other two; for the $\mathrm{V}$ component, two maxima are slightly higher than the third. These features are reproduced in the calculation in Fig. 3(a). So we attribute the terahertz emission from the substrate to bulk OR. The data from the layers, Fig. 2(b), do not show an azimuthal angle dependence due either to a pure bulk effect, Fig. 3(a), or a pure surface effect, Fig. 3(b). As for (211) InSb, ${ }^{19}$ a combination of these, Fig. 3(c), reproduces the experimental features well. We therefore conclude that the growth of the epilayer produces a surface electric field which enhances the emission of terahertz radiation. Comparing Figs. 2(a) and 2(b) it is seen the enhancement in the GaBiAs epilayer compared to the substrate is approximately threefold.

We conclude that TC effects play a negligible role in the emission of terahertz radiation from the GaBiAs epilayers studied. Rather, terahertz emission is due to a bulk OR enhanced by a surface OR in the epilayers.

We acknowledge the contribution of M. Shafi, R. H. Mari, and S. Novikov in preparing the samples. This work was supported by the Australian Research Council. We also acknowledge the UK EPSRC.

${ }^{1}$ K. Oe, Jpn. J. Appl. Phys., Part 1 41, 2801 (2002).

${ }^{2}$ M. Yoshimoto, S. Murata, A. Chayahara, Y. Horino, J. Saraie, and K. Oe, Jpn. J. Appl. Phys., Part 2 42, L1235 (2003).

${ }^{3}$ S. Tixier, M. Adamcyk, T. Tiedje, S. Francoeur, A. Mascarenhas, P. Wei, and F. Schiettekatte, Appl. Phys. Lett. 82, 2245 (2003).

${ }^{4}$ S. Francoeur, M.-J. Seong, A. Mascarenhas, S. Tixier, M. Adamcyk, and T. Tiedje, Appl. Phys. Lett. 82, 3874 (2003).

${ }^{5}$ B. Fluegel, S. Francoeur, A. Mascarenhas, S. Tixier, E. C. Young, and T. Tiedje, Phys. Rev. Lett. 97, 067205 (2006).

${ }^{6}$ D. G. Cooke, F. A. Hegmann, E. C. Young, and T. Tiedje, Appl. Phys. Lett. 89, 122103 (2006).

${ }^{7}$ V. Pačebutas, K. Bertulis, L. Dapkus, G. Aleksejenko, A. Krotkus, K. M. Yu, and W. Walukiewicz, Semicond. Sci. Technol. 22, 819 (2007).

${ }^{8}$ V. Pačebutas, K. Bertulis, G. Aleksejenko, and A. Krotkus, J. Mater. Sci.: Mater. Electron. 20, S363 (2009).

${ }^{9}$ G. Molis, R. Adomavičius, A. Krotkus, K. Bertulis, L. Giniūnas, J. Pocius, and R. Danielius, Electron. Lett. 43, 190 (2007).

${ }^{10}$ V. Pačebutas, A. Bičiūnas, K. Bertulis, and A. Krotkus, Electron. Lett. 44 1154 (2008)

${ }^{11}$ K. Bertulis, A. Krotkus, G. Aleksejenko, V. Pačebutas, R. Adomavičius, G. Molis, and S. Marcinkevičius, Appl. Phys. Lett. 88, 201112 (2006).

${ }^{12}$ M. Henini, J. Ibáñez, M. Schmidbauer, M. Shafi, S. V. Novikov, L. Turyanska, S. I. Molina, D. L. Sales, M. F. Chisholm, and J. Misiewicz, Appl. Phys. Lett. 91, 251909 (2007).

${ }^{13}$ S. Hargreaves and R. A. Lewis, Appl. Phys. Lett. 93, 242101 (2008).

${ }^{14}$ A. Rice, Y. Jin, X. F. Ma, X.-C. Zhang, D. Bliss, J. Larkin, and M. Alexander, Appl. Phys. Lett. 64, 1324 (1994).

${ }^{15}$ Y. H. Jin, X.-C. Zhang, and J. Nonlin, J. Nonlinear Opt. Phys. Mater. 4, 459 (1995).

${ }^{16}$ S. Kono, P. Gu, M. Tani, and K. Sakai, Appl. Phys. B: Lasers Opt. 71, 901 (2000).

${ }^{17}$ Q. Chen, M. Tani, Z. Jiang, and X.-C. Zhang, J. Opt. Soc. Am. B 18, 823 (2001).

${ }^{18}$ P. Gu, M. Tani, S. Kono, K. Sakai, and X.-C. Zhang, J. Appl. Phys. 91, 5533 (2002)

${ }^{19}$ V. L. Malevich, A. Krotkus, A. Bičiūnas, and V. Pačebutas, J. Appl. Phys. 104, 113117 (2008).

${ }^{20}$ M. Reid, I. V. Cravetchi, and R. Fedosejevs, Phys. Rev. B 72, 035201 (2005). 